Amiran Gogatishvili, A. Razmadze Mathematical Institute, Georgian Academy of Sciences, 1 M. Aleksidze St., Tbilisi 380093, Georgia. e-mail: gogati@rmi.acnet.ge Current address: Mathematical Institute, Academy of Sciences of the Czech Republic, Zitna 25, CZ 11567 Praha 1, Czech

Republic. e-mail: gogatish@math.cas.cz

\title{
FRACTIONAL MAXIMAL FUNCTIONS IN WEIGHTED BANACH FUNCTION SPACES
}

\author{
Abstract \\ We characterize the boundedness of fractional maximal functions \\ defined in general homogeneous type spaces from a weighted Banach \\ function spaces $(X, \sigma)$ into another $(Y, \omega)$.
}

\section{Introduction}

A homogeneous type space $(X, d, \mu)$ is a topological space with a complete measure $\mu$ such that compactly supported functions are dense in the space $L^{1}(X, \mu)$. Moreover, it is assumed that there is a nonnegative real-valued function $d: X \times X \rightarrow \mathbb{R}$ satisfying the following conditions:

(i) $d(x, x)=0$ for all $x \in X$;

(ii) $d(x, y)>0$ for all $x \neq y$ in $X$;

(iii) there is a constant $a_{0}$ such that $d(x, y) \leq a_{0} d(y, x)$ for all $x, y$ in $X$;

(iv) there is a constant $a_{1}$ such that $d(x, y) \leq a_{1}(d(x, z)+d(z, y))$ for all $x$, $y$ and $z$ in $X$;

(v) for each neighborhood $V$ of $x$ in $X$ there is $r>0$ such that the ball $B(x, r)=\{y \in X: d(x, y)<r\}$ is contained in $V$.

Furthermore the balls $B(x, r)$ are measurable for all $x$ and $r>0$ and there is a constant $b$ such that $\mu B(x, 2 r) \leq b \mu B(x, r)$ for all ball with $\mu B(x, r)>0$ (see [2], [3] and [20]).

For a locally summable function $f: X \rightarrow \mathbb{R}$ and a locally finite measure $\sigma$ the fractional maximal function is defined by

$$
M_{\gamma}(f d \sigma)(x)=\sup (\mu B)^{\gamma-1} \int_{B}|f(y)| d \sigma, 0 \leq \gamma<1,
$$

Key Words: Homogeneous type space, weighted Banach function space, fractional maximal function

Mathematical Reviews subject classification: 42B20, 42B25,46E30

Received by the editors November 16, 1998 
where the supremum is taken with respect to all balls $B$ of positive measure containing the point $x$.

Let $\sigma$ be a measure on $X$. A real normed linear space $Z=(Z, \sigma)$ of $\sigma-$ measurable functions $f$ on $X$ is said to be a Banach function space (BFS) if in addition to the usual norm axioms it satisfies the following properties:

1) the norm $\|f\|_{Z}=\|f\|_{Z, \sigma}$ is defined for every $\sigma$-measurable function $f$, and $f \in Z$ if and only if $\|f\|_{Z}<\infty$;

2) $\|f\|_{Z}=\||f|\|_{Z}$ for all $f \in Z$;

3) if $0 \leq f \leq g$ a.e., then $\|f\|_{Z} \leq\|g\|_{Z}$;

4) if $0 \leq f_{n} \uparrow f$ a.e., then $\left\|f_{n}\right\|_{Z} \uparrow\|f\|_{Z}$;

5) if $\sigma(E)<\infty$, then $\left\|\chi_{E}\right\|_{Z}<\infty$ (where $\chi_{E}$ is the characteristic function of the set $E)$;

6) if $\sigma(E)<\infty$, then $\int_{E} f(x) d \sigma \leq C_{E}\|f\|_{Z}$ for some constant $C_{E}, 0<$ $C_{E}<\infty$ independent of $f$.

Given a BFS $Z=(Z, \sigma)$, its associate space $Z^{\prime}=\left(Z^{\prime}, \sigma\right)$ given by

$$
Z^{\prime}=\left(Z^{\prime}, \sigma\right)=\left\{f: \int_{X} f(x) g(x) d \sigma<\infty \text { for every } g \in Z\right\}
$$

and endowed with the norm $\|f\|_{Z^{\prime}}=\sup \left\{\int_{X}|f(x) g(x)| d \sigma:\|g\|_{Z} \leq 1\right\}$ is also a Banach function space satisfying axioms 1)-6).

The spaces $Z, Z^{\prime}$ are complete normed linear spaces and $Z^{\prime \prime}=Z$. The Hölder inequality $\int_{X}|f(x) g(x)| d \sigma \leq\|f\|_{Z}\|g\|_{Z^{\prime}}$ holds for all $f \in Z$ and $g \in Z^{\prime}$ and is sharp. (See[1])

A function $f$ in a $\operatorname{BFS}(Z, \sigma)$ has absolutely continuous norm (AC norm), if $\left\|f \chi_{E_{k}}\right\|_{Z} \rightarrow 0$ for every sequence of set $\left\{E_{k}\right\} \subset X$ such, that $\chi_{E_{k}}(x) \rightarrow 0$ $\sigma-$ a.e.

The distribution function $\sigma_{f}$ of a $\sigma$ measurable function $f$ defined on $X$ is given by $\sigma_{f}(\lambda)=\sigma\{x \in X:|f|>\lambda\}, \lambda \geq 0$.

Two functions, a $\sigma$-measurable $f$ on $X$ and $\mu$-measurable $g$ on $Y$, are said to be equimeasurable if they have the same distribution function, that is, if $\sigma_{f}(\lambda)=\mu_{g}(\lambda)$ for all $\lambda \geq 0$.

Suppose $f$ is a $\sigma$-measurable function on $X$. The decreasing rearrangement of $f$ is the function $f_{\sigma}^{*}$ defined on $[0, \infty)$ by $f_{\sigma}^{*}(t)=\inf \left\{\lambda: \sigma_{f}(\lambda) \leq t\right\}$. As an immediate consequence, we get $f_{\sigma}^{*}(t)=\sup \left\{\lambda: \sigma_{f}(\lambda)>t\right\}=m_{\sigma_{f}}(t)$ where $m$ is Lebesgue measure on $[0, \infty)$. 
A Banach function space $Z$ is said to be a rearrangement-invariant space (r.i. BFS) if $\|f\|=\|g\|$ for every pair of equimeasurable functions $f$ and $g$ defined on $X$.

The fundamental function $\varphi_{Z}$ of a r.i. BFS $Z$ over $(X, \sigma)$ is defined for each finite value of $t$ belonging to the range of $\sigma$ by $\varphi_{Z}(t)=\left\|\chi_{E}\right\|_{Z}$ where $E$ is subset of $Z$ with $\sigma(E)=t$. (For more details we refer the the reader to [1])

Proposition 1.1. [1, Theorem 5.2] Let $(X, \sigma)$ be a nonatomic or a completely atomic measure space, with atoms having equal measure. Let $Z$ be a r.i. BFS over $(X, \sigma)$ and $Z^{\prime}$ its associate space of $Z$. Then $\varphi_{Z}(t) \varphi_{Z^{\prime}}(t)=t$ for each finite value of $t$ in the range of $\sigma$.

Proposition 1.2. [1, Corollary 5.3] Let $(X, \sigma)$ be a nonatomic or a completely atomic measure space, with atoms having equal measure. Let $Z$ be a r.i. BFS over $(X, \sigma)$. Then the fundamental function $\varphi_{Z}$ of $Z$ is increasing, $\varphi_{Z}(t)=0$ iff $t=0, \frac{\varphi_{Z}(t)}{t}$ is decreasing, $\varphi_{Z}$ is continuous, except perhaps at the origin.

Let $Z$ be a r.i. BFS over $(X, \sigma)$ with fundamental function $\varphi_{Z}$. Let $M_{Z}$ be the submultiplicative function $M_{Z}(t)=\sup _{s}\left\{\frac{\varphi_{Z}(s t)}{\varphi_{Z}(s)}\right\},(t>0)$, and define the lower and upper fundamental indices of $Z$ by

$$
\underline{\beta}_{Z}=\sup _{0<t<1} \frac{\log M_{Z}(t)}{\log t}, \bar{\beta}_{Z}=\inf _{t>1} \frac{\log M_{Z}(t)}{\log t} .
$$

(See in [1] and [19]) Then $0 \leq \underline{\beta}_{Z} \leq \bar{\beta}_{Z} \leq 1$ and $\bar{\beta}_{Z}=1-\underline{\beta}_{Z^{\prime}}$. We note that $\bar{\beta}_{Z}<1$ if and only if for some $\alpha<1$ there is a pair of positive constants $C$ and $\delta$ such that

$$
\frac{\varphi_{Z}(u)}{\varphi_{Z}(v)} \leq C\left(\frac{u}{v}\right)^{\alpha} \text { if } \frac{v}{u}<\delta
$$

while $\underline{\beta}_{Z}>0$ if and only if for some $\eta>0$ there is a pair of positive constant $C$ and $\delta$ such that

$$
\frac{\varphi_{Z}(v)}{\varphi_{Z}(u)} \leq C\left(\frac{v}{u}\right)^{\eta} \text { if } \frac{v}{u}<\delta
$$

Lemma 1.1. [19, Lemma 2.1] Let $(X, \sigma)$ be a nonatomic or a completely atomic measure space, with atoms having equal measure. Let $(Z, \sigma)$ be a r.i. BFS with fundamental function $\varphi_{Z}$ satisfying condition (1.1). Then for some constant $C(Z) \int_{0}^{t} \frac{1}{\varphi_{Z}(s)} d s \leq C(Z) \varphi_{Z^{\prime}}(t)$ for each finite value of $t$ in the range of $\sigma$ 
Lemma 1.2. [19, Lemma 3.1] Let $(X, \sigma)$ be a nonatomic or a completely atomic measure space, with atoms having equal measure. Let $Z$ be a r.i. BFS with upper fundamental index strictly less than 1 , and $1 \leq r<\infty$. Then

$$
\left(\int_{0}^{\infty}\left(\varphi_{Z}(\lambda) \frac{1}{\lambda} \int_{0}^{\lambda} f^{*}(s) d s\right)^{r} \frac{d \lambda}{\lambda}\right)^{\frac{1}{r}} \leq c\left(\int_{0}^{\infty}\left(\varphi_{Z}(\lambda) f^{*}(\lambda)\right)^{r} \frac{d \lambda}{\lambda}\right)^{\frac{1}{r}} .
$$

Definition 1.1 (15, definition 1.f.4). Let $1<p<\infty$. A BFS Z is said to satisfy an upper, resp. lower p-estimate, if for any sequence of disjoint measurable subsets $\left\{E_{i}\right\}$ and for all $f \in Z$ we have

$$
\left\|\sum_{i} \chi_{E_{i}} f\right\|_{Z} \leq d_{1}\left(\sum_{i}\left\|\chi_{E_{i}} f\right\|_{Z}^{p}\right)^{\frac{1}{p}} \text { resp. }\left\|\sum_{i} \chi_{E_{i}} f\right\|_{Z} \geq d_{1}^{-1}\left(\sum_{i}\left\|\chi_{E_{i}} f\right\|_{Z}^{p}\right)^{\frac{1}{p}}
$$

where $d_{1}$ is a finite positive constant independent of $f \in Z$ and $\left\{E_{i}\right\}$.

Lemma 1.3. [15, $p$. 83] Let $1<p<\infty$. A BFS Y satisfies an upper, respectively lower p-estimate if and only if $Y^{\prime}$ satisfies a lower, respectively upper $p^{\prime}$-estimate, where $p^{\prime}=\frac{p}{p-1}$.

Proposition 1.3. [15, proposition 1.f.6] Let $1<p<\infty$. A BFS Y satisfies a lower p-estimate if and only if for every choice of $\left\{f_{i}\right\}$ in $Y$ we have $\left\|\sum_{i}\left|f_{i}\right|\right\|_{Z} \geq d_{1}^{-1}\left(\sum_{i}\left\|f_{i}\right\|_{Z}^{p}\right)^{\frac{1}{p}}$.

Lemma 1.4. Let $(Z, \sigma)$ be a r.i. BFS satisfying an upper $p$-estimate $1<p$. Then the fundamental function $\varphi_{Z}$ satisfies condition (1.1).

Proof. Since $Z$ satisfies an upper p-estimate, $\varphi_{Z}\left(\sum_{i} t_{i}\right) \leq d_{1}\left(\sum_{i} \varphi_{Z}^{p}\left(t_{i}\right)\right)^{\frac{1}{p}}$ for every sequence $\left\{t_{i}\right\}$ in the range of $\sigma$. Therefore (see [8, Lemma 6.1.1] ) $\varphi_{Z}^{p}$ is concave and consequently $\frac{\varphi_{Z}^{p}(t)}{t}$ is decreasing and $\varphi_{Z}$ satisfies condition (1.1) with $\alpha=\frac{1}{p}$.

Next we assume that all BFS are defined over nonatomic measure or completely atomic measure spaces, with atoms having equal measure.

Throughout the paper the expressions of the form $0 \cdot \infty, 0 / 0$ and $\infty / \infty$ are taken to be equal to zero.

Main Theorem. Let $1<p<q<\infty, 0<\gamma<1$. Let $(Y, \omega)$ be a BFS satisfying an upper $q$ estimate and let $(Z, \sigma)$ be a r.i. BFS satisfying a lower p-estimate with upper fundamental index strictly less 1 . Then the following two conditions are equivalent: 
(i) there exists a constant $c_{1}>0$ such that for any $f \in Z$

$$
\left\|M_{\gamma}(f d \sigma)\right\|_{Y} \leq c_{1}\|f\|_{Z}
$$

(ii) there exists a constant $c_{2}>0$ such that for all balls $B(x, r) \subset X$

$$
\left\|\chi_{B(x, r)}\right\|_{Z^{\prime}}\left\|(\mu B(x, d(x, \cdot)+r))^{\gamma-1}\right\|_{Y} \leq c_{2} .
$$

For $Y=L^{p}(\omega)$ and $Z=L^{q}(\sigma) 1<p<q<\infty$ this was proved in [11]. For homogeneous type spaces having group structure it was first obtained in [21] (also see [18]). For Orlicz classes norm like inequalities were studied in [9]. For a detailed account of this subject see the book of I. Genebashvili , A.Gogatishvili, V.Kokilashvili and M.Krbec [8]. It should be noted that condition (1.4), which is a natural extension for BFS of a well known condition of M. Gabidzashvili, appears for the first time in $[5,13]$.

In section 2 we give another characterization of inequality (1.3) which is a generalization of a well known result of E. Sawyer [16], for Lebesgue spaces on Euclidean spaces and for homogeneous type spaces $[17,18]$. In section 3 we give several characterization of weak type inequalities in weighted BFS for integral transforms with positive kernels. In section 4 we give the proof of the main theorem which is based on results established in sections 2 and 3.

\section{Preliminary Results}

First we give a familiar covering lemma which is valid for arbitrary spaces with a quasi-metric.

Lemma A. [20, 8 lemma 1.3.1]. Let $E$ be a bounded set in $X$ and, for each point $x \in E$ let a ball $B_{x}=B\left(x, r_{x}\right)$ be given. Then from the family $\left\{B_{x}\right\}_{x \in E}$ we can choose a (finite or infinite) sequence of pairwise disjoint balls $\left(B_{j}\right)_{j}$ for which $E \subset \cup_{j \geq 1} N_{0} B_{j}, N_{0}=a_{1}\left(1+2 a_{0}\right)$, and for each $B_{x} \in\left\{B_{x}\right\}_{x \in E}$ there exists a ball $B_{j_{0}}$ such that $x \in N_{0} B_{j_{0}}$ and $\operatorname{rad} B_{x} \leq 2 \operatorname{rad} B_{j_{0}}$.

Lemma $\mathrm{A}$ is now used to help in the proof of the following.

Theorem 2.1. Let $(X, \sigma)$ be a nonatomic or a completely atomic measure space, with atoms having equal measure. Let $0 \leq \gamma<1,1<r<\infty$. Let $(Y, \omega)$ a BFS over $(X, \sigma)$ satisfying an upper $r$-estimate and $(Z, \sigma)$ a r.i. BFS over $(X, \sigma)$ satisfying a lower $r$-estimate with upper fundamental index strictly less than 1 . Then the following two conditions are equivalent:

(i) there exists a constant $c_{1}>0$ such that for any $f \in Z$

$$
\left\|M_{\gamma}(f d \sigma)\right\|_{Y} \leq c_{1}\|f\|_{Z}
$$


(ii) there exists a constant $c_{2}>0$ such that for all balls $B \subset X$

$$
\left\|M_{\gamma}\left(\chi_{B} d \sigma\right)\right\|_{Y} \leq c_{2}\left\|\chi_{B}\right\|_{Z} .
$$

Proof. On substituting $f=\chi_{B}$ in (2.1), we obtain (2.2). Therefore the implication $(i) \Rightarrow(i i)$ is fulfilled. We will prove $(i i) \Rightarrow(i)$.

By the principle of duality it is sufficient to show, that

$$
\int_{X} M_{\gamma}(f d \sigma)(x)|g(x)| d \omega \leq c\|f\|_{Z}\|g\|_{Y^{\prime}},
$$

for all $f \in Z$ and $g \in Y^{\prime}$. Let

$$
N=a_{1}\left(1+2 a_{1}\left(1+a_{0}\right)\right) \text { and } N_{1}=a_{1}\left(1+a_{1}^{2}\left(1+a_{0}\right)\left(N+a_{0}\right)\right) .
$$

Assume $b>2$ to be a constant such that $\mu\left(N_{1} B\right) \leq b \mu B$ for an arbitrary ball $B$. Further let $B_{0}$ be an arbitrarily fixed ball and $f$ an arbitrary integrable function which is nonnegative almost everywhere and satisfies the condition supp $f \subset B_{0}$. Following the ideas of [11], we set

$$
\Omega_{k}=\left\{x \in X: M_{\gamma}(f)(x)>b^{k}\right\}, \quad k \in \mathbb{Z} .
$$

Obviously, for each $x \in \Omega_{k}$ there exists a ball $B(y, r) \ni x$ such that

$$
\frac{1}{(\mu B(y, r))^{1-\gamma}} \int_{B(y, r)} f(z) d \sigma>b^{k} .
$$

The set of radii of such balls will be bounded by virtue of the fact that supp $f \subset$ $B_{0}$. Consider the values

$$
\mathcal{R}_{x}^{k}=\sup \left\{r: \exists B(y, r) \ni x, \frac{1}{(\mu B(y, r))^{1-\gamma}} \int_{B(y, r)} f(z) d \sigma>b^{k}\right\} .
$$

Obviously, for arbitrary $x \in \Omega_{k}$ there exist $y_{x} \in X$ and $r_{x}>\frac{\mathcal{R}_{x}^{k}}{2}$ such that

$$
\frac{1}{\left(\mu B\left(y_{x}, r_{x}\right)\right)^{1-\gamma}} \int_{B\left(y_{x}, r_{x}\right)} f(z) d \sigma>b^{k}
$$

Along with this, for each ball $B^{\prime}$ which contains the point $x$ and for which $\operatorname{rad} B^{\prime} \geq 2 r_{x}$ we have

$$
\frac{1}{\left(\mu B^{\prime}\right)^{1-\gamma}} \int_{B^{\prime}} f(z) d \sigma \leq b^{k}
$$


By Lemma A for each $k$ there exists a sequence $\left(B_{j}^{k}\right)_{j \geq 1}$ of nonintersecting balls such that $\cup_{j \geq 1} N B_{j}^{k} \supset \Omega_{k}$ and $\frac{1}{\left(\mu B_{j}^{k}\right)^{1-\gamma}} \int_{B_{j}^{k}} f(z) d \sigma>b^{k}$. Let us show that if $B_{j}^{k} \cap B_{i}^{n} \neq \varnothing$ and $n>k$, then

$$
N B_{i}^{n} \subset N B_{j}^{k} .
$$

To this end it will first be shown that if $r_{i}=\operatorname{rad} B_{i}, r_{j}=\operatorname{rad} B_{j}$, and $n>k$, then $r_{i}^{n}<\frac{r_{j}^{k}}{a_{1}\left(a_{0}+N\right)}$. Assume the opposite, i.e., $r_{j}^{k} \leq a_{1}\left(a_{0}+N\right) r_{i}^{n}$. Then for $y \in B_{j}^{k}$ and $x \in B_{j}^{k} \cap B_{i}^{n}$ we will have

$$
\begin{aligned}
d\left(x_{i}^{n}, y\right) & \leq a_{1}\left(d\left(x_{i}^{n}, x\right)+d(x, y)\right) \leq a_{1}\left(r_{i}^{n}+a_{1}\left(a_{0} d\left(x_{j}^{k}, x\right)+d\left(x_{j}^{k}, y\right)\right)\right) \\
& <a_{1}\left(r_{i}^{n}+a_{1}\left(1+a_{0}\right) r_{j}^{k}\right) \leq a_{1}\left(1+a_{1}^{2}\left(1+a_{0}\right)\left(N+a_{0}\right)\right) r_{i}^{n}=N_{1} r_{i}^{n} .
\end{aligned}
$$

where $x_{j}^{k}$ is the center of $B_{j}^{k}$. Therefore $B_{j}^{k} \subset N_{1} B_{i}^{n}$. Along with this, $2 r_{j}^{k}<N_{1} r_{i}^{n}$. Therefore by virtue of (2.3) we obtain

$$
b^{n}<\frac{1}{\left(\mu B_{i}^{n}\right)^{1-\gamma}} \int_{B_{i}^{n}} f(z) d \sigma \leq \frac{b}{\mu\left(N_{1} B_{i}^{n}\right)^{1-\gamma}} \int_{N_{1} B_{i}^{n}} f(z) d \sigma \leq b^{k+1} .
$$

Thus $n \leq k$, which leads to a contradiction. Therefore $r_{j}^{k}>a_{1}\left(a_{0}+N\right) r_{i}^{n}$. Now for $x \in N B_{i}^{n}$ and $y \in B_{i}^{n} \cap B_{j}^{k}$ we derive

$$
\begin{aligned}
d\left(x_{j}^{k}, x\right) & \leq a_{1}\left(d\left(x_{j}^{k}, y\right)+a_{1}\left(a_{0} d\left(x_{i}^{n}, y\right)+d\left(x_{i}^{n}, x\right)\right)\right) \\
& \leq a_{1}\left(r_{j}^{k}+a_{1}\left(a_{0}+N\right) r_{i}^{n}\right) \leq 2 a_{1} r_{j}^{k}<N r_{j}^{k} .
\end{aligned}
$$

Thus $N B_{i}^{n} \subset N B_{j}^{k}$ provided that $B_{j}^{k} \cap B_{i}^{n} \neq \varnothing$ and $n>k$.

We introduce the sets

$$
E_{j}^{k}=\left(N B_{j}^{k} \backslash \bigcup_{i=1}^{j-1} N B_{i}^{k}\right) \cap\left(\Omega_{k} \backslash \Omega_{k+1}\right), \quad k \in \mathbb{Z}, \quad j \in \mathbb{N} .
$$

and

$$
\Gamma_{l}=\left\{(k, j): b^{l}<\frac{1}{\sigma\left(N B_{j}^{k}\right)} \int_{B_{j}^{k}} f(x) d \sigma \leq b^{l+1}\right\}, \quad l \in \mathbb{Z} .
$$

As is easy to verify, $\bigcup_{j=1}^{\infty} E_{j}^{k}=\Omega_{k} \backslash \Omega_{k+1}$ and $E_{j}^{k} \cap E_{i}^{n}=\varnothing$ for $k \neq n$ or $i \neq j$. Therefore we have

$$
\int_{X} M_{\gamma}(f d \sigma)(x) g(x) d \omega \leq \sum_{k=-\infty}^{\infty} \sum_{j=1}^{\infty} b^{(k+1)} \int_{E_{j}^{k}} g(x) d \omega
$$




$$
\begin{aligned}
& \leq \sum_{l=-\infty}^{\infty} \sum_{(k, j) \in \Gamma_{l}} b \frac{1}{\left(\mu B_{j}^{k}\right)^{1-\gamma}} \int_{B_{j}^{k}} f(z) d \sigma \int_{E_{j}^{k}} g(x) d \omega \\
& \leq \sum_{l=-\infty}^{\infty} \sum_{(k, j) \in \Gamma_{l}} b^{l+2} \frac{\sigma\left(N B_{j}^{k}\right)}{\left(\mu B_{j}^{k}\right)^{1-\gamma}} \int_{E_{j}^{k}} g(x) d \omega .
\end{aligned}
$$

Now fix some $k_{0} \in \mathbb{Z}$ and $l \in \mathbb{Z}$ and consider the system of balls $\left(B_{j}^{k}\right)_{(j, k) \in \Gamma_{l}^{k_{0}}}$. where $\Gamma_{l}^{k_{0}}=\left\{(k, j) \in \Gamma_{l}: k>k_{0}\right\}$. Choose from the latter system a subsystem of nonintersecting balls in the following manner: take all balls of "rank" $k_{0}$, i.e., all balls $B_{j}^{k_{0}}, j=1,2, \ldots$ Pass to "rank" $k_{0}+1$. If some $B_{j}^{k_{0}+1}$ intersects with no $B_{j}^{k_{0}}$, then include it in the subsystem and otherwise discard. Next, compare the balls of rank $k_{0}+2$ with the ones already chosen in the abovedescribed manner and so on. We thus obtain the sequence of nonintersecting balls $\left\{\widetilde{B}_{i}\right\}_{i}$. According to (2.4), for every $(k, j) \in \Gamma_{l}^{k_{0}} N B_{j}^{k} \subset N \widetilde{B}_{i_{0}}$ for some $i_{0} \geq 1$, and therefore $\cup_{i=1}^{\infty} N \widetilde{B}_{i}=\cup_{(k, j) \in \Gamma_{l}^{k_{0}}} N B_{j}^{k}$. Let $\widetilde{E}_{i}=\bigcup_{N B_{j}^{k} \subset N \widetilde{B}_{i}} \quad E_{j}^{k}$ $N B_{j}^{k} \not \subset N \widetilde{B}_{l}, l<i$

and $E_{l}=\cup_{i=1}^{\infty} \widetilde{E}_{i}$. The family of sets $\left\{\widetilde{E}_{i}\right\}$ and $\left\{E_{l}\right\}$ are pairwise disjoint as $\left\{E_{j}^{k}\right\}$ is a pairwise disjoint family of sets. Hence we obtain

$$
\begin{aligned}
& \sum_{(k, j) \in \Gamma_{l}^{k_{0}}} b^{l+2} \frac{\sigma\left(N B_{j}^{k}\right)}{\left(\mu\left(B_{j}^{k}\right)\right)^{1-\gamma}} \int_{E_{j}^{k}} g(x) \omega \\
\leq & \sum_{i=1}^{\infty} \sum_{N B_{j}^{k} \subset N \widetilde{B}_{i}} c b^{l+2} \frac{\sigma\left(N B_{j}^{k}\right)}{\mu\left(N B_{j}^{k}\right)^{1-\gamma}} \int_{E_{j}^{k}} g(x) \omega \\
\leq & \sum_{i=1}^{\infty} \sum_{N B_{j}^{k} \subset N \widetilde{B}_{i}} \int_{E_{j}^{k}} b^{l+2} M_{\gamma}\left(\chi_{N \widetilde{B}_{i}} d \sigma\right)(x) g(x) d \omega(x) \\
\leq & \sum_{i=1}^{\infty} \int_{\widetilde{E}_{i}} c b^{l+2} M_{\gamma}\left(\chi_{N \widetilde{B}_{i}} d \sigma\right)(x) g(x) d \omega(x) \\
\leq & \sum_{i=1}^{\infty} c b^{l+2}\left\|M_{\gamma}\left(\chi_{N \widetilde{B}_{i}} d \sigma\right)(x)\right\|_{Y}\left\|\chi_{\widetilde{E}_{i}} g\right\|_{Y^{\prime}} \leq \sum_{i=1}^{\infty} c b^{l+2}\left\|\chi_{N \widetilde{B}_{i}}\right\|_{Z}\left\|_{\widetilde{E}_{i}} g\right\|_{Y^{\prime}} .
\end{aligned}
$$

Thus

$$
b^{l}<\frac{1}{\sigma\left(N \widetilde{B}_{i}\right)} \int_{\widetilde{B}_{i}} f(x) d \sigma \leq \frac{1}{\sigma\left(N \widetilde{B}_{i}\right)} \int_{\widetilde{B}_{i} \cap G(l)} f d \sigma+b^{l-1},
$$


where $G(l)=\left\{x: f(x)>b^{l-1}\right\}$. We have $b^{l-1}(b-1) \leq \frac{1}{\sigma\left(N \widetilde{B}_{i}\right)} \int_{\widetilde{B}_{i} \cap G(l)} f(x) d \sigma$. Therefore

$$
\sum_{i=1}^{\infty} \sigma\left(N \widetilde{B}_{i}\right) \leq b^{-l} \frac{b}{b-1} \sum_{i=1}^{\infty} \int_{\widetilde{B}_{i} \cap G(l)} f(x) d \sigma \leq b^{-l} \frac{b}{b-1} \int_{G(l)} f(x) d \sigma .
$$

Since $Z$ satisfies a lower $r$-estimate, Lemma 1.3 implies $Y^{\prime}$ satisfies a lower $r^{\prime}$-estimate By Hölder's inequality, the right-hand side of (2.8) is bounded by

$$
\begin{aligned}
& c b^{l+2}\left(\sum_{i=1}^{\infty}\left(\left\|\chi_{N \tilde{B}_{i}}\right\|_{Z}\right)^{r}\right)^{\frac{1}{r}}\left(\sum_{i=1}^{\infty}\left(\left\|\chi_{\widetilde{E}_{i}} g\right\|_{Y^{\prime}}\right)^{r^{\prime}}\right)^{\frac{1}{r^{\prime}}} \\
& \leq c b^{l+2} \varphi_{Z}\left(b^{-l} \frac{b}{b-1} \int_{G(l)} f(x) d \sigma\right)\left\|\chi_{E_{l}} g\right\|_{Y^{\prime}} .
\end{aligned}
$$

Here the constant $c$ does not depend on $k_{0}$. Now letting $k_{0} \rightarrow-\infty$ and using the Hölder inequality, we obtain from (2.5), (2.8) and the latter estimate that

$$
\int_{X} M_{\gamma}(f d \sigma)(x) g(x) d \omega \leq\left(\sum_{l=-\infty}^{\infty} c b^{(l+2) r} \varphi_{Z}^{r}\left(b^{-l} \frac{b}{b-1} \int_{G(l)} f(x) d \sigma\right)\right)^{\frac{1}{r}}\|g\|_{Y^{\prime}} .
$$

The sum in the right-hand side of (2.9) is bounded by

$$
\begin{aligned}
I & =\sum_{l=-\infty}^{\infty} c b^{(l+2) r} \varphi_{Z}^{r}\left(b^{-l} \frac{b}{b-1} \int_{G(l)} f(x) d \sigma\right) \\
& \leq \sum_{l=-\infty}^{\infty} c \int_{b^{l-2}}^{b^{l-1}} \varphi_{Z}^{r}\left(\frac{1}{t} \int_{\{x: f(x)>t\}} f(x) d \sigma\right) t^{r} \frac{d t}{t} \\
\leq & c \int_{0}^{\infty} \varphi_{Z}^{r}\left(\frac{1}{t} \int_{0}^{\sigma(\{x: f(x)>t\})} f^{*}(s) d s\right) t^{r} \frac{d t}{t} \\
= & c r \int_{0}^{\infty} \frac{\varphi_{Z}^{r}\left(\frac{1}{t} \int_{0}^{\sigma(\{x: f(x)>t\})} f^{*}(s) d s\right)}{\left(\frac{1}{t} \int_{0}^{\sigma(\{x: f(x)>t\})} f^{*}(s) d s\right)^{r}} \\
& \times \int_{0}^{\sigma(\{x: f(x)>t\})}\left(\frac{1}{t} \int_{0}^{\lambda} f^{*}(s) d s\right)^{r-1} f^{*}(\lambda) d \lambda t^{r-1} \frac{d t}{t} .
\end{aligned}
$$


Since $\frac{\varphi_{Z}(t)}{t}$ is a decreasing function, we get

$$
I \leq \frac{c}{r} \int_{0}^{\infty} \int_{0}^{\sigma(\{x: f(x)>t\})} \frac{\varphi_{Z}^{r}\left(\frac{1}{t} \int_{0}^{\lambda} f^{*}(s) d s\right)}{\frac{1}{t} \int_{0}^{\lambda} f^{*}(s) d s} f^{*}(\lambda) d \lambda t^{r-1} \frac{d t}{t}
$$

and the Fubini theorem yields

$$
I \leq c r \int_{0}^{\infty} \frac{f^{*}(\lambda)}{\int_{0}^{\lambda} f^{*}(s) d s} \int_{0}^{f^{*}(\lambda)} \varphi_{Z}^{r}\left(\frac{1}{t} \int_{0}^{\lambda} f^{*}(s) d s\right) t^{r} \frac{d t}{t} d \lambda .
$$

To estimate the inside integral we use (1.1), since $\bar{\beta}_{Z}$ is strictly less 1 . We can assume $\delta<1$. Then

$$
\begin{aligned}
I & \leq \frac{c C r}{\delta^{\alpha r}} \int_{0}^{\infty} \frac{f^{*}(\lambda)}{\int_{0}^{\lambda} f^{*}(s) d s} \varphi_{Z}^{r}\left(\frac{\delta}{f^{*}(\lambda)} \int_{0}^{\lambda} f^{*}(s) d s\right) f^{*}(\lambda)^{\alpha r} \int_{0}^{f^{*}(\lambda)} t^{(1-\alpha) r} \frac{d t}{t} d \lambda \\
& =\frac{c C r}{(1-\alpha) \delta^{\alpha r}} \int_{0}^{\infty} \frac{f^{*}(\lambda)}{\int_{0}^{\lambda} f^{*}(s) d s} \varphi_{Z}^{r}\left(\frac{\delta}{f^{*}(\lambda)} \int_{0}^{\lambda} f^{*}(s) d s\right) f^{*}(\lambda)^{r} d \lambda .
\end{aligned}
$$

As $\frac{\int_{0}^{\lambda} f^{*}(s) d s}{f^{*}(\lambda)} \geq \lambda$ and function $\frac{\varphi_{Z}(t)}{t}$ is decreasing we have

$$
I \leq c \int_{0}^{\infty}\left(\varphi_{Z}(\lambda) \frac{1}{\lambda} \int_{0}^{\lambda} f^{*}(s) d s\right)^{r} \frac{d \lambda}{\lambda} .
$$

Since upper fundamental index of $Z$ strictly less 1 using Lemma 1.2, we get

$$
I \leq c \int_{0}^{\infty}\left(\varphi_{Z}(\lambda) f^{*}(\lambda)\right)^{r} \frac{d \lambda}{\lambda} .
$$

Now we prove that

$$
\int_{0}^{\infty}\left(\varphi_{Z}(\lambda) f^{*}(\lambda)\right)^{r} \frac{d \lambda}{\lambda} \leq c\|f\|_{Z}^{r}
$$

Further as $Z$ satisfies a lower $r$-estimate we obtain

$$
\begin{aligned}
\int_{0}^{\infty}\left(\varphi_{Z}(\lambda) f^{*}(\lambda)\right)^{r} \frac{d \lambda}{\lambda} & \leq \sum_{k=-\infty}^{+\infty}\left(f^{*}\left(2^{k}\right)\right)^{r} \int_{2^{k}}^{2^{k+1}} \varphi_{Z}(\lambda)^{r} \frac{d \lambda}{\lambda} \\
& \leq c \sum_{k=-\infty}^{+\infty}\left(f^{*}\left(2^{k}\right)\right)^{r} \varphi_{Z}^{r}\left(2^{k}\right)
\end{aligned}
$$




$$
\begin{aligned}
& \leq c \sum_{k=-\infty}^{+\infty}\left(f^{*}\left(2^{k}\right)\right)^{r}\left\|\chi_{\left\{f^{*}\left(2^{k+1}\right)<f(x) \leq f^{*}\left(2^{k}\right)\right\}}\right\|_{Z}^{r} \\
& \leq c \sum_{k=-\infty}^{+\infty}\left\|f \chi_{\left\{f^{*}\left(2^{k+1}\right)<f(x) \leq f^{*}\left(2^{k}\right)\right\}}\right\|_{Z}^{r} \leq c\|f\|_{Z}^{r} ;
\end{aligned}
$$

i.e., (2.10) holds. Thus we have proved (2.1) for an arbitrary integrable function with compact support. Now let $f$ be an arbitrary function. By virtue of the foregoing arguments, for an arbitrary ball $B_{0}$ we have $\left.\| M_{\gamma}\left(\chi_{B_{0}} f d \sigma\right)(x)\right) \|_{Y} \leq$ $\left\|\chi_{B_{0}} f\right\|_{Z} \leq\|f\|_{Z}$. Letting rad $B_{0}$ tend to infinity, by the 4) we obtain (2.1).

\section{Weak Type Inequalities for Integral Transforms in Weighted BFS}

In this section we shall present theorems on weak type estimates in a weighted BFS for integral transforms with a positive kernel defined on homogeneous type spaces. Let $\sigma$ be a positive locally finite measure on $X$. Consider the operator

$$
K(f d \sigma)(x)=\sup _{t \geq 0}\left|\int_{X} k(x, y, t) f(y) d \sigma(y)\right|,
$$

where $K: X \times X \times[0, \infty) \rightarrow \mathbb{R}$ is a non-negative measurable kernel.

Theorem 3.1. Let $k: X \times X \times[0, \infty) \rightarrow \mathbb{R}$ be an arbitrary positive measurable kernel, let $\sigma$ and $\nu$ be positive locally finite measures on $X$ such that $\nu\{x\}=$ $\sigma\{x\}=0$ for any $x \in X$, let $(1<p<q<\infty)$, let $(Z, \sigma)$ be a BFS satisfying a lower $p$-estimate and let $(Y, \nu)$ be a BFS satisfying an upper q-estimate such that the characteristic function $\chi_{E}$ has $A C$ norm for every set $E$ of finite measure. If the condition

$$
c_{0}=\sup \left\|\chi_{B\left(x, 2 N_{0} r\right)}\right\|_{Y}\left\|\chi_{X \backslash B(x, r)} k(x, \cdot, t)\right\|_{Z^{\prime}}<\infty,
$$

where the supremum is taken over all $t \geq 0, r \geq 0$ and all $x \in X, N_{0}=$ $a_{1}\left(1+2 a_{0}\right)$, (the constants $a_{0}$ and $a_{1}$ are from the definition of a quasi-metric), then there exists a constant $c>0$ such that for any $f \in Z$ and arbitrary $\lambda>0$

$$
\lambda\left\|\chi_{\{x \in X: K(f d \sigma)(x)>\lambda\}}\right\|_{Y} \leq c\|f\|_{Z} .
$$

Proof. Let $f$ be an arbitrary nonnegative function from $(Z, \sigma)$ and $\lambda>$ 0 . Without loss of generality we assume that $\left\|\chi_{X}\right\|_{Y}^{-1}\|f\|_{Z}<\frac{\lambda}{2 c_{0}}$, since 
otherwise we will have $\left\|\chi_{X}\right\|_{Y}<\infty$ and

$$
\left\|\chi_{\{x \in X: K(f d \sigma)(x)>\lambda}\right\|_{Y} \leq\left\|\chi_{X}\right\|_{Y} \leq 2 c_{0} \lambda^{-1}\|f\|_{Z}
$$

and the theorem will be proved. Let $x \in E_{\lambda}=\{x \in X: K(f d \sigma)(x)>\lambda\}$. Then there exists $r_{0}>0$ depending on $x$ such that

$$
\left\|\chi_{B\left(x, N_{0} r_{0}\right)}\right\|_{Y}^{-1}\|f\|_{Z} \geq \frac{\lambda}{2 c_{0}}
$$

and

$$
\left\|\chi_{B\left(x, 2 N_{0} r_{0}\right)}\right\|_{Y}^{-1}\|f\|_{Z}<\frac{\lambda}{2 c_{0}} .
$$

One can easily verify that there exists $r_{0}>0$ for which (3.4) is fulfilled. However (3.4) cannot hold for any $r>0$, since in that case we will have

$$
\begin{aligned}
\int_{X \backslash B(x, r)} f(y) k(x, y, t) d \sigma & \leq\left\|\chi_{X \backslash B(x, r)} f\right\|_{Z}\left\|\chi_{X \backslash B(x, r)} k(x, \cdot, t)\right\|_{Z^{\prime}} \\
& \leq c_{0}\|f\|_{Z}\left\|\chi_{B(x, 2 N r)}\right\|_{Y}^{-1}<\frac{\lambda}{2} .
\end{aligned}
$$

If in the latter inequality we pass to the limit as $r \rightarrow 0$, then by virtue of the condition $\sigma\{x\}=0$ for arbitrary $x \in X$ we will obtain $K(f d \sigma)(x) \leq \frac{\lambda}{2}<\lambda$, which contradicts the condition $x \in E_{\lambda}$.

The above arguments imply in particular that there exists $r>0$ for which (3.3) holds. If we consider the supremum of such numbers $r$, then we will find $r_{0}>0$ for which both (3.3) and (3.4) are fulfilled. Then we obviously have

$$
\int_{X \backslash B\left(x, r_{0}\right)} f(y) k(x, y, t) d \sigma \leq c_{0}\|f\|_{Z}\left\|\chi_{B\left(x, 2 N r_{0}\right)}\right\|_{Y}^{-1}<\frac{\lambda}{2} .
$$

Hence for $x \in E_{\lambda}$ and the corresponding $r_{0}>0$ we obtain

$$
\sup _{t \geq 0} \int_{B\left(x, r_{0}\right)} f(y) k(x, y, t) d \sigma \geq \frac{\lambda}{2} .
$$

For each $k \in \mathbb{N}$ we put $r_{k}=\sup \left\{r:\left\|\chi_{B\left(x, N_{0} r\right)}\right\|_{Y} \leq 2^{-k}\left\|\chi_{B\left(x, N_{0} r_{0}\right)}\right\|_{Y}\right\}$. The sequence $\left(r_{k}\right)_{k \geq 0}$ thus chosen will be decreasing and tends to zero because $\nu\{x\}=0$ and the fact that characteristic function has AC norm. We have

$$
\left\|\chi_{B\left(x, N_{0} r_{k}\right)}\right\|_{Y} \leq 2^{-k}\left\|\chi_{B\left(x, N_{0} r_{0}\right)}\right\|_{Y} \leq\left\|\chi_{B\left(x, 2 N_{0} r_{k}\right)}\right\|_{Y} .
$$


Let $B_{k}=B\left(x, r_{k}\right), k \geq 0$. Using condition (3.1), inequality (3.5) and a chain of inequalities (3.6) we obtain

$$
\begin{aligned}
\frac{\lambda}{2} & \leq \sup _{t \geq 0} \int_{B\left(x, r_{0}\right)} f(y) k(x, y, t) d \sigma \\
& =\sup _{t \geq 0} \sum_{k=0}^{\infty} \int_{B\left(x, r_{k}\right) \backslash B\left(x, r_{k+1}\right)} f(y) k(x, y, t) d \sigma \\
& \leq \sup _{t \geq 0} \sum_{k=0}^{\infty}\left\|\chi_{B\left(x, r_{k}\right)} f\right\|_{Z}\left\|\chi_{X \backslash B\left(x, r_{k+1}\right)} k(x, \cdot, t)\right\|_{Z^{\prime}} \\
& \leq c_{0} \sum_{k=0}^{\infty}\left\|\chi_{B\left(x, r_{k}\right)} f\right\|_{Y}\left\|\chi_{B\left(x, 2 N_{0} r_{k+1}\right)}\right\|_{Y}^{-1} \\
& \leq c \sum_{k=0}^{\infty}\left\|\chi_{B\left(x, N_{0} r_{k}\right)}\right\|_{Y}^{\frac{q}{p}-1}\left\|\chi_{B\left(x, r_{k}\right)} f\right\|_{Z}\left\|\chi_{B\left(x, N_{0} r_{k}\right)}\right\|_{Y}^{-\frac{q}{p}} \\
& \leq c \sum_{k=0}^{\infty} 2^{-k\left(\frac{q}{p}-1\right)}\left\|\chi_{B\left(x, N_{0} r_{0}\right)}\right\|_{Y}^{\frac{q}{p}-1}\left\|\chi_{B\left(x, r_{k}\right)} f\right\|_{Z}\left\|\chi_{B\left(x, N_{0} r_{k}\right)}\right\|_{Y}^{-\frac{q}{p}} .
\end{aligned}
$$

Since it is assumed that $p<q<\infty$, we have $c_{p q}=\sum_{k=0}^{\infty} 2^{-k\left(\frac{q}{p}-1\right)}<\infty$. The latter chain of inequalities implies

$$
\begin{aligned}
& c_{p q}^{-1} \sum_{k=0}^{\infty} 2^{-k\left(\frac{q}{p}-1\right)} \frac{\lambda}{2} \\
\leq & c \sum_{k=0}^{\infty} 2^{-k\left(\frac{q}{p}-1\right)}\left\|\chi_{B\left(x, N_{0} r_{0}\right)}\right\|_{Y}^{\frac{q}{p}-1}\left\|\chi_{B\left(x, r_{k}\right)} f\right\|_{Z}\left\|\chi_{B\left(x, N_{0} r_{k}\right)}\right\|_{Y}^{-\frac{q}{p}} .
\end{aligned}
$$

Hence we conclude that there exist $n_{0}$ and $c>0$ such that

$$
\lambda \leq c\left\|\chi_{B\left(x, N_{0} r_{0}\right)}\right\|_{Y}^{\frac{q}{p}-1}\left\|\chi_{B\left(x, r_{n_{0}}\right)} f\right\|_{Z}\left\|\chi_{B\left(x, N_{0} r_{n_{0}}\right)}\right\|_{Y}^{-\frac{q}{p}} .
$$

Taking (3.3) into account in the latter inequality we obtain

$$
\lambda \leq c \lambda^{1-\frac{q}{p}}\|f\|_{Z}^{\frac{q}{p}-1}\left\|\chi_{B\left(x, r_{n_{0}}\right)} f\right\|_{Z}\left\|\chi_{B\left(x, N_{0} r_{n_{0}}\right)}\right\|_{Y}^{-\frac{q}{p}}
$$

which implies $\left\|\chi_{B\left(x, N_{0} r_{n_{0}}\right)}\right\|_{Y} \leq c \lambda^{-1}\left\|\chi_{B\left(x, r_{n_{0}}\right)} f\right\|_{Z}^{\frac{p}{q}}\|f\|_{Z}^{1-\frac{p}{q}}$. To summarize the obtained results, we conclude that for each $x \in E_{\lambda}$ there exists a ball $B_{x}$ centered at the point $x$ such that

$$
\left\|\chi_{N_{0} B_{x}}\right\|_{Y} \leq c \lambda^{-1}\left\|\chi_{B_{x}} f\right\|_{Z}^{\frac{p}{q}}\|f\|_{Z}^{1-\frac{p}{q}}
$$


Since (3.7) holds for any $x \in E_{\lambda}$, the family $\left\{B_{x}\right\}_{x \in E_{\lambda}}$ covers the set $E_{\lambda}$. Let $B_{0}$ be an arbitrary ball in $X$. By Lemma A from the family $\left\{B_{x}\right\}$ we can choose a sequence of nonintersecting balls $\left(B_{k}\right)_{k}$ such that $E_{\lambda} \cap B_{0} \subset \cup_{k \geq 1}^{\infty} N_{0} B_{k}$. Using that $Y$ satisfies an upper $q$-estimate and $Z$, a lower $p$-estimate, (3.7) implies

$$
\begin{aligned}
\left\|\chi_{E_{\lambda} \cap B_{0}}\right\|_{Y} & \leq c\left(\sum_{k}\left\|\chi_{N_{0} B_{k}}\right\|_{Y}^{q}\right)^{\frac{1}{q}} \\
& \leq c \lambda^{-1}\left(\sum_{k}\left\|\chi_{B_{k}} f\right\|_{Z}^{p}\right)^{\frac{1}{q}}\|f\|_{Z}^{1-\frac{p}{q}} \leq c \lambda^{-1}\|f\|_{Z}^{\frac{p}{q}}\|f\|_{Z}^{1-\frac{p}{q}}
\end{aligned}
$$

and consequently $\left\|\chi_{E_{\lambda} \cap B_{0}} f\right\|_{Y} \leq c \lambda^{-1}\|f\|_{Z}$ where the constant $c$ does not depend on $B_{0}, \lambda$ and $f$. If in the latter inequality we pass to the limit as $\operatorname{rad} B_{0} \rightarrow \infty$, we will find that inequality (3.2) is valid.

Theorem 3.2. Let $1<p<q<\infty$ and $(Z, \sigma)$ and $(Y, \nu)$ be BFS satisfying the conditions of Theorem 3.1. Let $k: X \times X \times[0, \infty) \rightarrow \mathbb{R}$ be a positive measurable kernel such that there exists a constant $c_{1}$ with

$$
k(a, y, t) \leq c_{1} k(x, y, t)
$$

for arbitrary $t \geq 0, a \in X, y \in X \backslash B(a, r), r>0$ and $x \in B\left(a, 2 N_{0} r\right)$. Then conditions (3.1) and (3.2) are equivalent.

Proof. The implication (3.1) $\Rightarrow$ (3.2) follows from Theorem 3.1 without condition (3.8).

Let us prove the implication $(3.2) \Rightarrow(3.1)$. First it will be shown that for any $x \in X, r>0$ and $t \geq 0$ we have

$$
\left\|\chi_{X \backslash B(x, r)} k(x, \cdot, t)\right\|_{Z^{\prime}}<\infty .
$$

Assume to the contrary that for some $a \in X, r>0$ and $t_{0} \geq 0$ we have $\left\|\chi_{X \backslash B(a, r)} k\left(a, \cdot, t_{0}\right)\right\|_{Z^{\prime}}=\infty$. Then by duality there exists nonnegative $g$ : $X \rightarrow \mathbb{R}$ such that $\left\|\chi_{X \backslash B(a, r)} g\right\|_{Z} \leq 1$ and $\int_{X \backslash B(a, r)} g(y) k\left(a, y, t_{0}\right) d \sigma=+\infty$. On the other hand, by condition (3.8) we have

$T(g d \sigma)(x) \geq \int_{X \backslash B(a, r)} g(y) k\left(x, y, t_{0}\right) d \sigma \geq c^{\prime} \int_{X \backslash B(a, r)} g(y) k\left(a, y, t_{0}\right) d \sigma=+\infty$.

for arbitrary $x \in B(a, r)$. Thus $B(a, r) \subset\{x \in X: T(g d \sigma)(x)>\lambda\}$ for arbitrary $\lambda>0$. Thus by (3.2) we get $\left\|\chi_{B(a, r)}\right\|_{Y} \leq\left\|\chi_{\{x \in X: T(g d \sigma)(x)>\lambda}\right\|_{Y} \leq c \lambda^{-1}$. 
Recalling that $\lambda$ is an arbitrary positive number, from the latter inequality we conclude that $\left\|\chi_{B(a, r)}\right\|_{Y}=0$ and by 6) $\mu(B(a, r))=0$, which is impossible. Therefore (3.9) holds. Now we can proceed proving the implication $(3.2) \Rightarrow(3.1)$.

Let $B(x, r)$ be an arbitrary ball and $z \in B\left(x, 2 N_{0} r\right)$. By condition (3.8) we have

$T(f d \sigma)(z) \geq \int_{X \backslash B(x, r)} f(y) k(z, y, t) d \sigma \geq \frac{1}{c_{1}} \int_{X \backslash B(x, r)} f(y) k(x, y, t) d \sigma, t \geq 0$,

for an arbitrary nonnegative function $f: X \rightarrow \mathbb{R}$. Therefore from (3.2) we derive the inequality

$$
\begin{aligned}
\left\|\chi_{B\left(x, 2 N_{0} r\right)}\right\|_{Y} & \leq\left\|\chi_{\left\{z \in X: T(f d \sigma)(z)>\frac{1}{2 c_{1}} \int_{X \backslash B(x, r)} f(y) k(x, y, t) d \sigma\right.}\right\|_{Y} \\
& \leq 2 c_{1} c\left(\int_{X \backslash B(x, r)} f(y) k(x, y, t) d \sigma\right)^{-1}\|f\|_{Z},
\end{aligned}
$$

where the constants on the right-hand side do not depend on $x \in X, r>0$ and $t \geq 0$. In the latter inequality taking the supremum over all $f$ such that $\|f\|_{Z} \leq 1$ we obtain

$$
\left\|\chi_{B\left(x, 2 N_{0} r\right)}\right\|_{Y}\left\|\chi_{X \backslash B(x, r)} k(x, \cdot, t)\right\|_{Z^{\prime}} \leq c
$$

where the constant does not depend on $x \in X, r>0$ and $t \geq 0$. The latter implies that condition (3.1) is fulfilled.

In the theorems proved above our consideration is limited to spaces for which $\nu\{x\}=\sigma\{x\}=0$ for any $x \in X$. Below we will treat a more general case.

Theorem 3.3. Let $k: X \times X \times[0, \infty) \rightarrow \mathbb{R}$ be an arbitrary positive measurable kernel. Let $\sigma$ and $\nu$ be positive locally finite measures on $X$ having only isolated atoms. Let $(Z, \sigma)$ be a BFS satisfying a lower p-estimate and $(Y, \nu)$ a BFS satisfying an upper $q$-estimate such that the characteristic function $\chi_{E}$ has $A C$ norm for every set $E$ of finite measure, $(1<p<q<\infty)$. Suppose

$$
\sup _{\substack{t>0 \\ x \in X \\ \mu\{x\}>0}} k(x, x, t)\left\|\chi_{\{x\}}\right\|_{Y}\left\|\chi_{\{x\}}\right\|_{Z^{\prime}}<\infty
$$

is fulfilled along with (3.1). Then the conclusion of Theorem 3.1 is valid, i.e., (3.2) holds. 
Proof. After analyzing Theorem 3.1 we find that in the general case inequality (3.7) holds for any $x \in E_{\lambda}$ for which (3.3) and (3.4) are fulfilled simultaneously. For the case $\sigma\{x\}>0$ inequality (3.4) may hold for arbitrary $r>0$. Nevertheless it will be shown below that inequality (3.7) remains valid for the general case too. Let $x \in E_{\lambda}, \sigma\{x\}>0$ and $f(x) \sigma\{x\} \sup _{t \geq 0} k(x, x, t)<\frac{\lambda}{2}$. Since then we have

$$
\lim _{r \rightarrow 0} \int_{B(x, r)} f(y) k(x, y, t) d \sigma=f(x) k(x, x, t) \sigma\{x\},
$$

for each $t \geq 0$ there will exist $r_{t}$ such that

$$
\int_{B\left(x, r_{t}\right)} f(y) k(x, y, t) d \sigma<\frac{\lambda}{2}
$$

Let us show that in that case inequality (3.4) cannot be fulfilled for arbitrary $r>0$. Indeed, if this is so, then by virtue of $(3.1),(3.4)$ and (3.11) we will have

$$
\begin{aligned}
T(f d \sigma)(x) & \leq \sup _{t \geq 0} \int_{B\left(x, r_{t}\right)} f(y) k(x, y, t) d \sigma+\sup _{t \geq 0} \int_{X \backslash B\left(x, r_{t}\right)} f(y) k(x, y, t) d \sigma \\
& \leq \sup _{t \geq 0} \int_{B\left(x, r_{t}\right)} f(y) k(x, y, t) d \sigma+\sup _{t \geq 0}\left\|\chi_{X \backslash B\left(x, r_{t}\right)} k(x, \cdot, t)\right\|_{Z^{\prime}}\|f\|_{Z} \\
& \leq \sup _{t \geq 0} \int_{B\left(x, r_{t}\right)} f(y) k(x, y, t) d \sigma+c_{0} \sup _{t \geq 0}\left\|\chi_{B\left(x, 2 N_{0} r_{t}\right)}\right\|^{-1}\|f\| \\
& \leq \sup _{t \geq 0} \int_{B\left(x, r_{t}\right)} f(y) k(x, y, t) d \sigma+\frac{\lambda}{2} \leq \lambda
\end{aligned}
$$

which contradicts $x \in E_{\lambda}$. The contradiction obtained shows that in the case under consideration (3.4) cannot be fulfilled for any $r>0$ and therefore, in common with the proof of Theorem 3.1, we can find $r>0$ such that (3.3) and (3.4) will be fulfilled simultaneously, which fact leads to (3.7).

Assuming now that $x \in E_{\lambda}, \sigma\{x\}>0$ and $f(x) \sigma\{x\} \sup _{t \geq 0} k(x, y, t) \geq \frac{\lambda}{2}$, we obtain

$$
\begin{aligned}
\lambda & \leq 2 f(x) \sigma\{x\} \sup _{t \geq 0} k(x, x, t) \\
& \leq 2 f(x))\left\|\chi_{\{x\}}\right\|_{Y}^{-1}\left\|\chi_{\{x\}}\right\|_{Z}\left\|\chi_{\{x\}}\right\|_{Y} \sup _{t \geq 0} k(x, x, t)\left\|\chi_{\{x\}}\right\|_{Z^{\prime}} .
\end{aligned}
$$

By (3.10) the latter inequality gives rise to

$$
\left\|\chi_{\{x\}}\right\|_{Y} \leq c \lambda^{-1} f(x)\left\|\chi_{\{x\}}\right\|_{Z} .
$$


Since $\sigma\{x\}>0$, the point $x$ is isolated. Therefore there exists $r>0$ such that $B\left(x, N_{0} r\right)=\{x\}$. Now (3.12) can be rewritten as

$$
\left\|\chi_{B\left(x, N_{0} r\right)}\right\|_{Y} \leq c \lambda^{-1}\left\|\chi_{B\left(x, N_{0} r\right)} f\right\|_{Z} \leq c \lambda^{-1}\left\|\chi_{B\left(x, N_{0} r\right)} f\right\|_{Z}^{\frac{p}{q}}\|f\|_{Z}^{1-\frac{p}{q}}
$$

which implies that (3.7) is valid. Thus for all $x \in E_{\lambda}, \nu\{x\}>0$ there exists a ball $B_{x}$ centered at the point $x$ such that (3.7) is fulfilled. In common with the proof of Theorem 3.1 we conclude that (3.2) is valid.

Theorem 3.4. Let $1<p<q<\infty$ and $(Z, \sigma)$ and $(Y, \nu)$ be BFS satisfying conditions of Theorem 3.3. Let $k: X \times X \times[0, \infty) \rightarrow \mathbb{R}$ be a positive measurable kernel satisfying the condition (3.8). Then the inequality (3.2) is equivalent to the set of conditions (3.1) and (3.10).

Proof. As shown in the proof of the preceding theorem, conditions (3.1) and (3.10) imply inequality (3.2) without condition (3.8). By the proof of Theorem 3.2 it remains to show that $(3.2) \Rightarrow(3.10)$. Let $\sigma\{x\}>0$. It is easy to verify that $T(f d \sigma)(x)>\frac{1}{2} f(x) k(x, x, t) \sigma\{x\}, t \geq 0$. Therefore

$$
\{x\} \subset\left\{y \in X: T(f)(y)>\frac{1}{2} f(x) k(x, x, t) \sigma\{x\}\right\} .
$$

By the latter inclusion and condition (3.2) we obtain

$$
\begin{aligned}
\left\|\chi_{\{x\}}\right\|_{Y} & \leq\left\|\chi_{\left\{y \in X: T(f d \sigma)(y)>\frac{1}{2} f(x) k(x, x, t) \sigma\{x\}\right.}\right\|_{Y} \\
& \leq 2 c(f(x) k(x, x, t) \sigma\{x\})^{-1}\|f\|_{Z} .
\end{aligned}
$$

After taking the supremum over all $f$ such that $\|f\|_{Z} \leq 1$ we obtain

$$
\left\|\chi_{\{x\}}\right\|_{Y} k(x, x, t)\left\|\chi_{\{x\}}\right\|_{Z^{\prime}} \leq c .
$$

Since the constant $c$ does not depend on $x$ and $t$, from the latter inequality we conclude that condition (3.10) is fulfilled.

The analysis of the above theorems gives rise to the following two remarks:

Remark 3.1. When $X=\mathbb{R}^{n}$ and $d$ is Euclidean distance, we can take the constant $N_{0}$ in the above theorems to be 1 . This can be done because the Besicovitch covering lemma (see $[12,13]$ ) can be applied instead of Lemma A to Euclidean spaces. 
Remark 3.2. When $X=\mathbb{R}^{n}$ and space the $(Y, \nu)$ is such that $\left\|\chi_{B(x, r)}\right\|_{Y}$ is continuous with respect to $r$, we can replace $\left\|\chi_{B\left(x, 2 N_{0} r\right)}\right\|_{Y}$ in condition (3.1) by $\left\|\chi_{B(x, r)}\right\|_{Y}$ and, accordingly, weaken condition (3.8) in Theorem 3.2 to: there exists a constant $c_{1}>0$ such that $k(a, y, t) \leq c_{1} k(x, y, t)$ for arbitrary $t \geq 0, a, x$ and $y$ from $X$ satisfying $d(a, x) \leq d(a, y)$.

Next we will consider the case where $k(x, y, t) \equiv k(x, y)$. It will again be assumed that the measure $\sigma$ is locally finite. Let

$$
\mathcal{K}(f d \sigma)(x)=\int_{X} k(x, y) f(y) d \sigma, \text { and } \mathcal{K}^{*}(f d \sigma)(x)=\int_{X} k(y, x) f(y) d \sigma .
$$

We assume that if $f$ is nonnegative, then for all $\lambda>0$, all sets of the forms $\{x \in X: \mathcal{K}(f d \sigma)(x)>\lambda\}$ are open.

Definition 3.1. A positive measurable kernel $k: X \times X \rightarrow \mathbb{R}$ is said to satisfy condition $(V)(k \in V)$ if there exists a constant $c>0$ such that $k(x, y)<$ $c k\left(x^{\prime}, y\right)$ for arbitrary $x, y$ and $x^{\prime}$ from $X$ such that $d\left(x, x^{\prime}\right)<N d(x, y)$, where $N=2 N_{0}$.

Theorem 3.5. Let $1<p<q<\infty$. Let $\sigma$ and $\nu$ be positive locally finite measures on $X$ having only isolated atoms, let $(Z, \sigma)$ be a BFS satisfying a lower p-estimate, let $(Y, \nu)$ be a r.i. BFS satisfying an upper q-estimate such that the characteristic function $\chi_{E}$ has $A C$ norm for every set $E$ of finite measure and let $k: X \times X \rightarrow \mathbb{R}$ be a positive measurable kernel satisfying the condition $(V)$. Then the following conditions are equivalent:

i) there exists a constant $c_{1}>0$ such that for arbitrary $\lambda>0$ and nonnegative $f \in(Z, \sigma)$

$$
\left\|\chi_{\{x \in X: \mathcal{K}(f d \sigma)(x)>\lambda\}}\right\|_{Y} \leq c_{1} \lambda^{-1}\|f\|_{Z}
$$

ii) there exists a constant $c_{2}>0$ such that for an arbitrary ball $B \subset X$

$$
\left\|\mathcal{K}^{*}\left(\chi_{B} d \nu\right)(x)\right\|_{Z^{\prime}} \leq c_{2}\left\|\chi_{B}\right\|_{Y^{\prime}}
$$

iii)

$$
\sup _{\substack{x \in X \\ r>0}}\left\|\chi_{B\left(x, 2 N_{0} r\right)}\right\|\left\|_{Y}\right\| \chi_{X \backslash B(x, r)} k(x, \cdot) \|_{Z^{\prime}}<\infty
$$


Proof. The implication i) $\Leftrightarrow$ iii) follows from Theorem 3.2. We will prove ii) $\Rightarrow$ iii). Applying the condition $(V)$ for the kernel for any $y \in X \backslash B(x, r)$, we obtain

$$
\mathcal{K}^{*}\left(\chi_{B\left(x, 2 N_{0} r\right)} d \nu\right)(y)=\int_{B\left(x, 2 N_{0} r\right)} k(z, y) d \nu \quad \geq c^{-1} k(x, y) \nu\left(B\left(x, 2 N_{0} r\right)\right) .
$$

By the latter inequality and $((3.14))$ using Proposition 1.1 we conclude that (3.15) holds.

Finally, it will be shown that the implication i) $\Rightarrow$ ii) is valid. We have $\left\|\mathcal{K}^{*}\left(\chi_{B} d \nu\right)\right\|_{Z^{\prime}}=\sup \int_{X} \mathcal{K}^{*}\left(\chi_{B} d \nu\right)(x) g(x) d \sigma$, where the exact upper bound is taken with respect to all $g$ for which $\|g\|_{Z} \leq 1$. By Fubini's theorem $\int_{X} \mathcal{K}^{*}\left(\chi_{B} d \nu\right)(x) g(x) d \sigma=\int_{B} \mathcal{K}(g d \sigma)(y) d \nu$. If we take $\lambda=(\mathcal{K}(g d \sigma))_{\nu}^{*}(t)$ by definition of the decreasing rearrangement from inequality (3.13) we get that $(\mathcal{K}(g d \sigma))_{\nu}^{*}(t) \leq \frac{c}{\varphi_{Y}(t)}$. As $Y$ satisfies an upper $q$-estimate $(1<q<\infty)$, by Lemma 1.4 the fundamental function $\varphi_{Y}$ satisfies (1.1) and using Lemma 1.1 and Proposition 1.1 we get

$$
\begin{aligned}
\int_{B} \mathcal{K}(g d \sigma)(y) d \nu & =\int_{0}^{\infty}\left(\chi_{B} \mathcal{K}(g d \sigma)\right)_{\nu}^{*}(t) d t \\
& \leq c_{1} \int_{0}^{\nu B} \frac{1}{\varphi_{Y}(t)} d t \leq c_{2} \varphi_{Y^{\prime}}(\nu B)=c_{2}\left\|\chi_{B}\right\|_{Y^{\prime}}
\end{aligned}
$$

and thereby prove the implication i) $\Rightarrow$ ii) and, accordingly, the theorem.

In [6], [7] and [10] the two-weight problem was solved for integral transforms with a positive kernel in Lebesgue spaces, in [14] for Lorentz spaces and in [9] for Orlicz classes (also see [4], [18, [22] and [8]).

In the rest of this section we no longer assume that $\mu$ satisfies the doubling condition. We will assume that for the space $X$ there is a number $L>1$ such that any ball $B(x, r)$ contains at most $L$ points $x_{i}$ with $d\left(x_{i}, x_{m}\right)>\frac{r}{2}$. The assumption that a measure $\mu$ given in $X$ satisfies the doubling condition guarantees the fulfillment of the above-formulated condition (see [2], Lemma 1.1). For such space $X$ the following statements are valid.

Lemma B. Let $\Omega$ be an open subset of $X$ and $c \geq 1$. Then there exists a sequence of balls $B_{j}=B\left(x_{j}, r_{j}\right)$ such that

(i) $\Omega=\bigcup_{j=1}^{\infty} \bar{B}_{j}=\bigcup_{j=1}^{\infty} B_{j}$, where $\bar{B}_{j}=B\left(x_{j}, c r_{j}\right)$;

(ii) there exists $M=M\left(c, L, a_{0}, a_{1}\right)>0$ such that $\sum_{j=1}^{\infty} \chi_{\bar{B}_{j}}(x) \leq M$;

(iii) $(X \backslash \Omega) \cap \overline{\bar{B}}_{j} \neq \varnothing$ for each $\overline{\bar{B}}_{j}=B\left(x_{j}, 3 c a_{1} r_{j}\right)$. 
This Lemma was formulated for bounded set in ([3], p. 623), but it is valid for any open set.

Theorem 3.6. Let $\sigma$ and $\nu$ be positive locally finite measures on $X$ let $(Z, \sigma)$ be a BFS satisfying a lower p-estimate $1<p<\infty$, let $(Y, \nu)$ be a r.i. BFS satisfying an upper $p$-estimate with lower fundamental index strictly greater than 0 and let $k: X \times X \rightarrow \mathbb{R}$ be a positive measurable kernel satisfying $(V)$. Then the following conditions are equivalent:

i) there exists a constant $c_{1}>0$ such that for arbitrary $\lambda>0$ and nonnegative $f \in(Z, \sigma)$

$$
\left\|\chi_{\{x \in X: \mathcal{K}(f d \sigma)(x)>\lambda\}}\right\|_{Y} \leq c_{1} \lambda^{-1}\|f\|_{Z}
$$

ii) there exists a constant $c_{2}>0$ such that for an arbitrary ball $B \subset X$

$$
\left\|\mathcal{K}^{*}\left(\chi_{B} d \nu\right)(x)\right\|_{Z^{\prime}} \leq c_{2}\left\|\chi_{B}\right\|_{Y^{\prime}} .
$$

Proof. Let $f$ be a bounded nonnegative function with compact support. For given $\lambda>0$ we set $\Omega_{\lambda}=\{x \in X: \mathcal{K}(f d \sigma)(x)>\lambda\}$. Let $B_{j}=B\left(x_{j}, r_{j}\right)$ be the sequence of balls from Lemma B for the number $c=2 a_{1}$. Then there exists a constant $c_{3}>0$ such that (see, for instance, [16]) $\mathcal{K}\left(\chi_{X \backslash c B_{j}} f \sigma\right)(x) \leq c_{3} \lambda$ for any $x \in B_{j}$, where $c B_{j}=B\left(x_{j}, c r_{j}\right)$. Hence $\mathcal{K}\left(\chi_{c B_{j}} f \sigma\right)(x)>c_{3} \lambda$ for any $x \in B_{j} \cap \Omega_{2 c_{3} \lambda}$. Then,

$$
\nu \Omega_{2 c_{3} \lambda} \leq \sum_{j=1}^{\infty} \nu\left(B_{j} \cap \Omega_{2 c_{3} \lambda}\right)=\sum_{j \in F}+\sum_{j \in G} \nu\left(B_{j} \cap \Omega_{2 c_{3} \lambda}\right)=I_{1}+I_{2},
$$

where $F=\left\{j: \nu\left(B_{j} \cap \Omega_{2 c_{3} \lambda}\right)>\varepsilon \nu\left(c B_{j}\right)\right\}, G=\left\{\nu\left(B_{j} \cap \Omega_{2 c_{3} \lambda}\right) \leq \varepsilon \nu\left(c B_{j}\right)\right\}$, and the number $\varepsilon$ will be chosen below so that $0<\varepsilon<1$.

Applying the Fubini theorem, the Hölder inequality and condition (3.17) we obtain for $j \in F$

$$
\begin{gathered}
c_{3} \lambda \nu\left(c B_{j}\right) \leq c_{3} \varepsilon^{-1} \lambda \nu\left(B_{j} \cap \Omega_{2 c_{3} \lambda}\right) \leq \varepsilon^{-1} \int_{B_{j}}\left(\int_{c B_{j}} k(x, y) f(y) d \sigma(y)\right) d \nu(x) \\
\leq \varepsilon^{-1} \int_{c B_{j}}\left(\int_{B_{j}} k(x, y) d \nu(x)\right) f(y) d \sigma(y)=\varepsilon^{-1} \int_{c B_{j}} \mathcal{K}^{*}\left(\chi_{B_{j}} d \nu\right)(y) f(y) d \sigma \\
\leq \varepsilon^{-1}\left\|\mathcal{K}^{*}\left(\chi_{c B_{j}} d \nu\right) \chi_{c B_{j}}\right\|_{Z^{\prime}}\left\|\chi_{c B_{j}} f\right\|_{Z} \leq \varepsilon^{-1} c_{2}\left\|\chi_{c B_{j}}\right\|_{Y^{\prime}}\left\|\chi_{c B_{j}} f\right\|_{Z} .
\end{gathered}
$$

Therefore $\lambda \nu\left(c B_{j}\right) \leq c_{3}^{-1} \varepsilon^{-1} c_{2}\left\|\chi_{c B_{j}}\right\|_{Y^{\prime}}\left\|\chi_{c B_{j}} f\right\|_{Z}$. The summation of the latter inequality, applying Hölder's inequality, the fact that $Z$ satisfies a lower 
$p$-estimate, $Y$ satisfies an upper $p$-estimate and, by Lemma 1.4, $Y^{\prime}$ satisfies a lower $p^{\prime}$-estimate and $\sum_{j=1}^{\infty} \chi_{c B_{j}} \leq M$, gives

$$
I_{1} \leq \lambda^{-1} c_{4}\left(\sum_{j=1}^{\infty}\left\|\chi_{c B_{j}}\right\|_{Y^{\prime}}^{p^{\prime}}\right)^{\frac{1}{p^{\prime}}}\left(\sum_{j=1}^{\infty}\left\|\chi_{c B_{j}} f\right\|_{Z}^{p}\right)^{\frac{1}{p}} \leq \lambda^{-1} c_{5}\left\|\chi_{\Omega_{2 c_{3} \lambda}}\right\|\left\|_{Y^{\prime}}\right\| f \|_{Z} .
$$

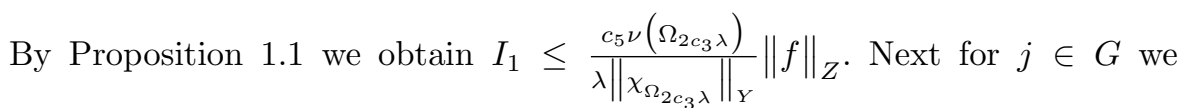
obtain $\nu\left(B_{j} \cap \Omega_{2 c_{3} \lambda}\right) \leq \varepsilon \nu\left(c B_{j}\right)$. Therefore $I_{2} \leq \varepsilon M \nu \Omega_{\lambda}$. Finally, since the lower fundamental index $\underline{\beta}_{Y}>0, \varphi_{Y}$ satisfies (1.2) and therefore we find that $\lambda\left\|\chi_{\Omega_{2 c_{3} \lambda}}\right\|_{Y} \leq c_{5}\|f\|_{Z}+\varepsilon^{\eta} M \lambda\left\|\chi_{\Omega_{2 c_{3} \lambda}}\right\|_{Y}$. Taking the exact upper bound with respect to $\lambda, 0<\lambda<\frac{s}{2 c_{3}}$, we obtain $\sup _{0<\lambda<s} \lambda\left\|\chi_{\Omega_{\lambda}}\right\|_{Y} \leq c_{5}\|f\|_{Z}+$ $\varepsilon^{\eta} M \sup _{0<\lambda<s} \lambda\left\|\chi_{\Omega_{\lambda}}\right\|_{Y}$. If we set $\varepsilon=\frac{1}{2 M\left(2 c_{3}\right)^{\frac{1}{\eta}}}$, the latter inequality will imply $\sup _{0<\lambda<s} \lambda\left\|\chi_{\Omega_{\lambda}}\right\|_{Y} \leq c_{5}\|f\|_{Z}+\frac{1}{2} \sup _{0<\lambda<s} \lambda\left\|\chi_{\Omega_{\lambda}}\right\|_{Y}$. If the second term on the right-hand side is assumed to be finite, then from the latter inequality we obtain $\sup _{0<\lambda<s} \lambda\left\|\chi_{\Omega_{\lambda}}\right\|_{Y} \leq 2 c_{5}\|f\|_{Z}$ Letting $s$ tend to infinity, we concluded that (3.16) holds.

It remains to show that for arbitrary finite $s$

$$
\sup _{0<\lambda<s} \lambda\left\|\chi_{\Omega_{\lambda}}\right\|_{Y}<\infty
$$

Let supp $f \subset B$, where $B=B\left(x_{0}, r\right)$ is a ball in $X$. It is obvious that $\lambda\left\|\chi_{B\left(x_{0}, 2 a_{1} r\right)}\right\|_{Y} \leq s\left\|\chi_{B\left(x_{0}, 2 a_{1} r\right)}\right\|_{Y}<\infty$. Therefore it is sufficient to show that $\sup _{\lambda>0} \lambda\left\|\chi_{\Omega_{\lambda} \backslash B\left(x_{0}, 2 a_{1} r\right)}\right\|_{Y}<\infty$. Let $x \in \Omega_{\lambda} \backslash B\left(x_{0}, 2 a_{1} r\right)$ and $B_{x}=$ $B\left(x_{0}, 2 d\left(x_{0}, x\right)\right)$. For $x^{\prime} \in B_{x}$ and $y \in B$ we have $2 a_{1} d\left(x_{0}, y\right) \leq d\left(x_{0}, x\right) \leq$ $a_{1}\left(d\left(x_{0}, y\right)+d(y, x)\right)$. Hence $d\left(x_{0}, y\right) \leq a_{0} d(x, y)$. Further for $\overline{x^{\prime}} \in B_{x}$ and $y \in B$ we have

$$
\begin{aligned}
d\left(x^{\prime}, y\right) & \leq a_{1}\left(d\left(x^{\prime}, x_{0}\right)+d\left(x_{0}, y\right)\right) \leq a_{1} a_{0} d(x, y)+2 a_{1} a_{0} d\left(x_{0}, x\right) \\
& \leq a_{1} a_{0} d(x, y)+2 a_{1}^{2} a_{0}\left(d\left(x_{0}, y\right)+d(y, x)\right) \\
& \leq a_{1} a_{0} d(x, y)+4 a_{1}^{2} a_{0}^{2} d(x, y)=\left(a_{1} a_{0}+4 a_{1}^{2} a_{0}^{2}\right) d(x, y) .
\end{aligned}
$$

Now by virtue of the remark made at the beginning of the proof of Proposition 3.1 from [10] there exists a constant $c^{\prime}$ such that $k(x, y) \leq c^{\prime} k\left(x^{\prime}, y\right)$ for any $x^{\prime} \in B_{x}$ and $y \in B$. Therefore using (3.17) and the Hölder inequality we obtain

$$
\lambda \nu\left(B_{x}\right) \leq \nu\left(B_{x}\right) \int_{B} k(x, y) f(y) d \sigma(y) \leq\left\|\chi_{B_{x}}\right\|_{Z}\left\|\chi_{B_{x}} k(x, \cdot) \nu\left(B_{x}\right)\right\|_{Z^{\prime}}
$$




$$
\leq c_{5}\left\|\chi_{B_{x}}\right\|_{Z}\left\|\chi_{B} \mathcal{K}^{*}\left(\chi_{B_{x}} d \nu\right)\right\|_{Z^{\prime}} \leq c_{6}\left\|\chi_{B_{x}}\right\|_{Z}\left\|\chi_{B_{x}}\right\|_{Y^{\prime}} .
$$

Hence by Proposition 1.1 we conclude that $\lambda\left\|\chi_{B_{x}}\right\|_{Y} \leq c_{6}\left\|\chi_{B_{x}}\right\|_{Z}$ Since $\Omega_{\lambda} \backslash B\left(x_{0}, 2 a_{1} r\right) \subset \cup B_{x}$ and balls $B_{x}$ have a common center, by virtue of the axiom 6$)$ the latter estimate implies that (3.18) is valid. Thus the implication $(3.17) \Rightarrow(3.16)$ is proved for functions $f$ with a compact support. One can easily pass over to the general case.

The implication $(3.16) \Rightarrow(3.17)$ is proved by Theorem 3.5 .

Theorem 3.6 for Lebesgue spaces was proved in [11] with another proof is in [22]. For Orlicz classes It was established in [9], (also see [8]).

\section{Proof of the Main Theorem}

Using the results of the preceding sections we will prove the main theorem of this paper.

Proof of the Main Theorem. Our aim is to show that the implication $(1.3) \Leftrightarrow(1.4)$ is valid. First we will prove the implication $(1.4) \Rightarrow(1.3)$. Consider an operator on $(Z, \sigma)$ in the form $T_{\gamma}(f d \omega)(x)=\int_{X} \frac{|f(y)|}{(\mu B(x, d(x, y)))^{1-\gamma}} d \omega$. The latter operator is an analog of the Riesz potential for homogeneous type spaces. that

First note that condition (1.4) implies that there exists a constant $c$ such

$$
\sup \left\|\chi_{B\left(x, 2 N_{0} r\right)}\right\|_{Z^{\prime}} \| \chi_{X \backslash B(x, r)}\left(\mu B(x, d(x, \cdot))^{\gamma-1} \|_{Y}<c,\right.
$$

for all $x \in X$ and $r>0$. Indeed by the doubling condition for $y \in X \backslash B(x, r)$ we have that

$$
\mu B\left(x, d(x, y)+2 N_{0} r\right) \leq \mu B\left(x,\left(2 N_{0}+1\right) d(x, y)\right) \leq b^{\prime} \mu B(x, d(x, y))
$$

and therefore

$$
\begin{aligned}
& \left\|\chi_{B\left(x, 2 N_{0} r\right)}\right\|_{Z^{\prime}} \| \chi_{X \backslash B(x, r)}\left(\mu B(x, d(x, \cdot))^{\gamma-1} \|_{Y}\right. \\
& \leq b^{\prime}\left\|\chi_{B\left(x, 2 N_{0} r\right)}\right\|_{Z^{\prime}} \|\left(\mu B \left(x, d\left(x, d(x, \cdot)+2 N_{0} r\right)^{\gamma-1} \|_{Y} \leq b^{\prime} c_{2} .\right.\right.
\end{aligned}
$$

By Lemma $1.3\left(Z^{\prime}, \sigma\right)$ is a r.i. BFS satisfying an upper $p^{\prime}$-estimate and $\left(Y^{\prime}, \omega\right)$ is a BFS satisfying a lower $q^{\prime}$-estimate. As $q^{\prime}<p^{\prime}$ when $p<q$, using Theorem 3.5, for $\left(Z^{\prime}, \sigma\right)$ and $\left(Y^{\prime}, \omega\right)$ we conclude that $\left\|\chi_{\left\{x \in X: T_{\gamma}(f d \omega)(x)>\lambda\right\}}\right\|_{Z^{\prime}} \leq$ $c_{3} \lambda^{-1}\|f(x)\|_{Y^{\prime}}$ with the constant $c_{3}$ not depending on $\lambda>0$ and $f$ is valid.

Further, again by virtue of Theorem 3.5 the latter inequality implies that there exists a constant $c_{4}>0$ such that for any ball $B \subset X$ we have

$$
\left\|T_{\gamma}^{*}\left(\chi_{B} \sigma\right)\right\|_{Y} \leq c_{4}\left\|\chi_{B}\right\|_{Z}
$$


where $T_{\gamma}^{*}(\varphi \sigma)(x)=\int_{X} \frac{|\varphi(y)|}{(\mu B(y, d(x, y)))^{1-\gamma}} d \sigma$.

On the other hand, there exist constants $c_{3}>0$ and $c_{4}>0$ such that

$$
c_{3} \mu B(y, d(x, y)) \leq \mu B(x, d(x, y)) \leq c_{5} \mu B(y, d(x, y)) .
$$

The latter follows from the fact that $B(x, d(x, y)) \subset B\left(a_{1}\left(a_{0}+1\right) d(x, y)\right)$. Indeed, let $d(x, z) \leq d(x, y)$. Then $d(y, z) \leq a_{1}(d(y, x)+d(x, z)) \leq a_{1}\left(a_{0}+\right.$ 1) $d(x, y)$. By virtue of the doubling property for measure we obtain

$$
\mu B\left(y, a_{1}\left(a_{0}+1\right) d(x, y)\right) \leq c_{6} \mu B(y, d(x, y)) .
$$

Hence we conclude that (4.2) is valid. Next, from (4.2) and (4.1) we derive

$$
\left\|T_{\gamma}\left(\chi_{B} \sigma\right)\right\|_{Y} \leq c_{7}\left\|\chi_{B}\right\|_{Z}
$$

Now use inequality (see in [10]) $M_{\gamma}(\varphi \sigma)(x) \leq c_{7} T_{\gamma}(\varphi \sigma)(x)$, from (4.3) we obtain $\left\|M_{\gamma}\left(\chi_{B} \sigma\right)\right\|_{Y} \leq c_{8}\left\|\chi_{B}\right\|_{Z}$. By Theorem 2.1 we conclude that inequality (1.3) is valid. Thus we have proved the implication (1.4) $\Rightarrow(1.3)$. Let us show the validity of the inverse implication $(1.3) \Rightarrow(1.4)$. Fix an arbitrary ball $B(x, r)$ and assume $f(y)=\chi_{B(x, r)}(y)$. Obviously we have

$$
M_{\gamma}(f \sigma)(y) \geq \frac{\sigma(B(x, d(x, y)+r) \cap B(x, r))}{\mu B(x, d(x, y)+r)^{1-\gamma}} \geq \frac{\sigma(B(x, r))}{\mu B(x, d(x, y)+r)^{1-\gamma}} .
$$

Therefore (1.4) implies

$$
\sigma(B(x, r))\left\|\mu B(x, d(x, \cdot)+r)^{\gamma-1}\right\|_{Y} \leq \leq c\left\|\chi_{B(x, r)}\right\|_{Z} .
$$

From the latter inequality using Proposition 1.1 we obtain (1.4).

Definition 4.1. The measure $\nu$ satisfies the reverse doubling condition $(\nu \in$ $(R D))$ if there exist numbers $\delta$ and $\varepsilon$ from $(0,1)$ such that $\nu B\left(x, r_{1}\right) \leq$ $\varepsilon \nu B\left(x, r_{2}\right)$ for $\mu B\left(x, r_{1}\right) \leq \delta \mu B\left(x, r_{2}\right), 0<r_{1}<r_{2}$.

In the particular case where measure $\sigma$ satisfies the reverse doubling condition, (1.4) in the main theorem can be replaced by a simpler condition.

Theorem 4.1. Let $1<p<q<\infty, 0<\gamma<1$, let $(Y, \omega)$ be a BFS satisfying an upper q-estimate, let $(Z, \sigma)$ be a r.i. BFS satisfying a lower p-estimate with upper fundamental index strictly less than 1 and let measure $\sigma$ satisfy the reverse doubling condition. Then (1.3) holds iff

$$
\sup _{\substack{x \in X \\ r>0}}(\mu B(x, r))^{\gamma-1}\left\|\chi_{B(x, r)}\right\|\left\|_{Z^{\prime}}\right\| \chi_{B(x, r)} \|_{Y}<\infty .
$$


Proof. The implication (1.3) $\Rightarrow(4.4)$ is obtained immediately by substituting the function $f(y)=\chi_{B}(y)$ into (1.3).

By the main theorem, to prove the implication $(4.4) \Rightarrow(1.3)$ it is sufficient to show that if $\sigma \in(R D)$, then (4.4) $\Rightarrow(1.4)$. Let $x \in X$ and $r>0$ be fixed. Choose numbers $r_{k}(k=0,1, \ldots)$ such that $r_{0}=r$ and by induction $r_{k}=\inf \left\{r: \mu B\left(x, r_{k-1}\right)<\delta \mu B(x, r)\right\}$. Obviously, $\mu B\left(x, r_{k-1}\right)<$ $\delta \mu B\left(x, r_{k}\right)<c \mu B\left(x, r_{k-1}\right)$. Again applying the condition $(R D)$, we obtain $\sigma B\left(x, r_{0}\right) \leq \varepsilon^{k} \sigma B\left(x, r_{k}\right) \quad k=1,2, \ldots$. Since the upper fundamental index of $Z$ is strictly less than 1 , that $\varphi_{Z}$ satisfies (1.2) and on using this the latter inequalities imply

$$
\begin{aligned}
\| \chi_{B(x, r)} & \left\|_{Z^{\prime}}\right\|(\mu B(x, d(x, \cdot)+r))^{\gamma-1}\left\|_{Y} \leq\right\| \chi_{B(x, r)}\left\|_{Z^{\prime}}(\mu B(x, r))^{\gamma-1}\right\| \chi_{B(x, r)} \|_{Y} \\
& +\left\|\chi_{B\left(x, r_{0}\right)}\right\|_{Z^{\prime}} \sum_{k=1}^{\infty}\left\|\chi_{B\left(x, r_{k}\right) \backslash B\left(x, r_{k-1}\right)}(\mu B(x, d(x, \cdot)))^{\gamma-1}\right\|_{Y} \\
\leq & c \sum_{k=0}^{\infty} \varphi_{Z^{\prime}}\left(\varepsilon^{k} \sigma B\left(x, r_{k}\right)\right)\left\|\chi_{B\left(x, r_{k}\right)}\right\|_{Y}\left(\mu B\left(x, d\left(x, r_{k}\right)\right)\right)^{\gamma-1} \\
\leq & c \sum_{k=0}^{\infty} \varepsilon^{\alpha k} \varphi_{Z^{\prime}}\left(\sigma B\left(x, r_{k}\right)\right)\left\|\chi_{B\left(x, r_{k}\right)}\right\|_{Y}\left(\mu B\left(x, d\left(x, r_{k}\right)\right)\right)^{\gamma-1} \\
\leq & c \sum_{k=0}^{\infty} \varepsilon^{\alpha k}\left(\mu B\left(x, d\left(x, r_{k}\right)\right)\right)^{\gamma-1}\left\|\chi_{B\left(x, r_{k}\right)}\right\|_{Z^{\prime}}\left\|\chi_{B\left(x, r_{k}\right)}\right\|_{Y} \\
\leq & c \sum_{k=0}^{\infty} \varepsilon^{\alpha k}<\infty .
\end{aligned}
$$

Therefore (1.3) holds. This completes the proof of Theorem 4.1.

For Lebesgue spaces this theorem was proved in [11] and for Orlicz classes, in [9]. (See also [8], [18] and [21])

Acknowledgment. I wish to express my gratitude to the Royal Society and NATO for support under their Postdoctoral Fellowship program to visit the School of Mathematics at Cardiff during 1997/98. I also thank the Georgian Academy of Sciences for partial support under grant No. 1.7 of the Georgian Academy of Sciences.

\section{References}

[1] C. Bennett and R. Sharpley, Interpolation of operators, Pure Appl. Math. 129, Academic Press, 1988 
[2] R. R. Coifman and G. Weiss, Analyse harmonique non-commutative sur certains espaces homogenes, Lecture Notes in Math. 242, Springer-Verlag, Berlin and New York, 1971.

[3] R. R. Coifman and G. Weiss, Extensions of Hardy spaces and their use in Analysis, Bull. Amer. Math. Soc., 83 (1977), 569-645.

[4] B. Franchi, C. Gutiérrez and R. Wheeden, Weighted Sobolev-Poincaré inequalities for Grushin type operators, Comm. P.D.E., 19 (1994), 523604 .

[5] M. Gabidzashvili, Weighted inequalities for anisotropic potentials, (Russian), Trudy Tbil. Mat. Inst. Razmadze Akad. Nauk Gruz. SSR, 89 (1986), 25-36.

[6] I. Genebashvili, A. Gogatishvili, and V. Kokilashvili, Solution of twoweight problems for integral transforms with positive kernels, Georg. Math. J., 3 (1996), no. 4, 319-34.

[7] I. Genebashvili, A. Gogatishvili, and V. Kokilashvili, Criteria of twoweighted inequalities for integral transforms with a positive kernel and maximal functions, Dokl. Akad. Nauk RAN, 351 (1996) no. 4, 448-451.

[8] I. Genebashvili, A. Gogatishvili,V. Kokilashvili and M. Krbec, Weight theory for integral transforms on spaces of homogeneous type, Pitman Monographs and Surveys in Pure and Applied Mathematics, 92 Longman, Harlow, 1998.

[9] A. Gogatishvili, Two-weight mixed inequalities in Orlicz classes for fractional maximal functions defined on homogeneous type spaces, Proc. A. Razmadze Math. Inst., 112 (1997), 23-56.

[10] A. Gogatishvili and V. Kokilashvili, Criteria of weight inequalities for integral transforms defined on homogeneous type spaces. In: Topological Vector Spaces, Algebras and Related Areas, Longman. Pitman Research Notes in Mathematics 316 (1994), 251-262.

[11] A. Gogatishvili and V. Kokilashvili, Criteria of strong type two-weighted inequalities for fractional maximal functions, Georgian Math. J., 3 (1996), no. 5, 423-446.

[12] M. de Guzmán, A covering lemma with applications to differentiability of measures and singular integral operators, Studia Math., 34 (1970), 299-317. 
[13] V. Kokilashvili and M. Gabidzashvili, Two-weight weak type inequalities for fractional type integrals, Preprint No. 45, Math. Inst. Czech. Akad. Sci., Praha, 1989.

[14] V. Kokilashvili, Riesz potentials in weighted Lorentz spaces. In: Continuum Mechanics and Related Problems in Analysis, Proc. Int. Symp., Metsniereba, Tbilisi 1993, 382-389.

[15] J. Lindenstrauss and L. Tzafriri, Classical Banach spaces. II, Springer Verlag, New York, 1979

[16] E. T. Sawyer, A characterization of a two-weight norm inequality for maximal operators, Studia Math., 75 (1982), 1-11.

[17] E. T. Sawyer and R. L. Wheeden, Weighted inequalities for fractional integrals on Euclidean and homogeneous type spaces, Amer. J. Math., 114 (1992), 813-875.

[18] E. T. Sawyer, R. L. Wheeden and S. Zhao, Weighted norm inequalities for operators of potential type and fractional maximal functions, Potential Analysis, 5 (1996), 523-580.

[19] R. Sharpley, Spaces $\Lambda_{\alpha}(X)$ and interpolation, J. Functional Anal., 11 (1972), 479-513.

[20] J. O. Strömberg and A. Torchinsky, Weighted Hardy spaces, Lect. Notes in Math., 1381, Springer Verlag, New York, 1989.

[21] R. L. Wheeden, A characterization of some weighted norm inequalities for fractional maximal functions, Studia Math., 107 (1993), 251-272.

[22] R. L. Wheeden and S. Zhao, Weak type estimates for operators of potential type, Studia Math., 119 (1994), 149-160. 